\title{
200 años del ingreso de José Gervasio Artigas al Paraguay
}

Fecha de Recepción: 12 de Julio de 2020

Fecha de Aceptación: 20 de Septiembre de 2020

DOI: https://doi.org/10.46553/RGES.56.2020.p.157-183

En este 2020 se cumplen doscientos años de la llegada de José Gervasio Artigas al Paraguay, país en el que vivió durante treinta años hasta su fallecimiento, en 1850. Por tal motivo, el suplemento cultural del diario $A B C$ Color convocó a cuatro historiadores de Uruguay, Argentina y Paraguay para escribir sobre el contexto y las circunstancias en que se produjo ese hecho, y ofrecer observaciones que permitieran arrojar luz sobre las tres décadas que Artigas pasó en el Paraguay, siendo testigo de profundas transformaciones políticas y sociales. Durante la preparación de los textos los autores dialogamos, intercambiamos ideas, nos leímos y nos hicimos más conscientes -si cabe- sobre la necesidad de profundizar la conversación. De este modo, nos pareció oportuno revisar y ampliar los márgenes de las contribuciones para luego publicarlas en el formato de una auténtica mesa redonda de diálogo e intercambio de informaciones sobre el exilio, la vida social en el Paraguay decimonónico y las conexiones del federalismo en Uruguay, Paraguay y Argentina.

\section{José Artigas y el nacimiento del federalismo correntino}

\section{Dardo Ramírez Braschi*}

Los aniversarios, en ocasiones, actúan como disparadores que nos llevan a recordar y repensar sucesos históricos que sellan la vida política de los Estados y, en ese marco, el rol que desempeñaron algunos hombres determinantes en las construcciones políticas. Este es el caso de José Gervasio de Artigas, que lo rememoramos en ocasión de cumplirse dos siglos de su arribo al Paraguay.

El siglo XIX trajo como impronta en la región del Río de la Plata el surgimiento de nuevos Estados, que inmediatamente entraron en la encrucijada de la organización

\footnotetext{
*Universidad Nacional del Nordeste, dramirezbraschi@ yahoo.com.ar
} 
institucional, cuestión ésta de fortísimas consecuencias. Una de las cuestiones a resolver era la manera en que el poder se distribuiría en los espacios territoriales y si cada una de las fragmentaciones territoriales serían poseedoras de derechos y podrían ser ejercidas autonómicamente.

Ante estos asuntos, tempranamente surgieron posiciones que reivindicarían la soberanía de los pueblos, tal como lo hizo Paraguay y, poco tiempo después, las provincias litorales bajo la protección del artiguismo.

Así surgieron las primeras soberanías, que no solamente bregaron por la independencia, sino también por un orden político equitativo, lo que llevaría a asumir posiciones de defensa de derechos soberanos que visiblemente se mantendrían durante la segunda década, bajo la impronta del artiguismo: construir un Estado desde la organización confederativa-federativa (en los momentos iniciales no se apreciaban marcadas diferencias de lo que cada uno de estos conceptos significaba).

En esta ocasión, quisiera exponer los ligámenes de los principios del artiguismo con la evolución del Estado Provincial correntino, sus ideales republicanos y el concepto de federación, que caracterizarán toda la etapa preconstituyente.

Ya en 1814, en pleno accionar de la Asamblea Constituyente de Buenos Aires reunida a partir de 1813-, la situación en Corrientes llegaó a una tensa inquietud tras la necesidad de discutir los principios que los diputados reunidos en Buenos Aires omitían reconocer: la administración de los derechos de las provincias. En el tratamiento de esta cuestión, dos serán los hitos fundacionales en la voluntad de los correntinos: la disposición Capitular del 20 de abril de 1814 y el Congreso Constituyente de junio de 1814.

El Cabildo correntino dejó para la posteridad el Acta del 20 de abril de 1814, donde expresaba su adhesión al artiguismo, comulgando con las ideas del caudillo oriental al declarar la Independencia bajo el sistema federativo, a pesar de que sectores capitulares opondrían resistencia. Pero la representación de la Sala Capitular no contaba con una legitimidad que aglutinase a todos los pueblos adherentes a la ciudad, razón por lo cual el artiguismo consideraría indispensable la necesidad de convocar a un Congreso, con la representatividad de todos los pueblos de la jurisdicción correntina. Entonces, el Acta Capitular del 20 de abril de 1814 representa la primera manifestación, expresa y formal, de adhesión de Corrientes al sistema federal de gobierno, revelando sus derechos autonómicos al "declarar la Independencia de la Provincia bajo el sistema federativo". ${ }^{1}$

\footnotetext{
${ }^{1}$ Archivo General de la Provincia de Corrientes. Actas Capitulares, Tomo 46 - Fs. 1 (vta.).
} 
Artigas creía indispensable que las provincias, a través de sus propios órganos políticos, tomasen las decisiones necesarias encaminadas a la libertad e independencia, afianzando sus potestades políticas, tal como lo demuestran las correspondencias que intercambiaba con el gobernador de Corrientes Juan Bautista Méndez, en cuyas líneas el caudillo oriental declaraba que se tornaba imprescindible ir avanzando en la organización del país, constituyendo una Liga con las demás provincias. ${ }^{2}$

El segundo momento destacado en esta cuestión emergió dos meses después, más precisamente el 11 de junio, cuando se dieron inicio las sesiones del Congreso, conformado por representantes de toda la provincia. ${ }^{3}$ En el seno del Congreso las ideas y los proyectos a seguir no fueron unánimes. Había disparidad en los criterios, y la principal dificultad por resolver gravitaba alrededor de la intensidad del vínculo con Buenos Aires. En esa intensidad residía la diferencia. Uno de los grupos, cuyos voceros principales eran José Simón García de Cossio y Ángel Fernández Blanco, buscaba incorporar a Corrientes al orden institucional vinculado con la Asamblea constituyente que sesionaba en Buenos Aires.

Sujetarse a Buenos Aires implicaba entrar vigorosamente en la órbita de sus decisiones, y, por lo tanto, suponía una adhesión incondicional a la política del Directorio, la que estaba muy distante de reconocer principios de soberanía o algún accionar de autonomía por parte de las provincias. Más aún, los partidarios de esta política encontraron el rostro visible para enfrentar militarmente al artiguismo: Genaro Perugorría. Oportunamente, éste sería representante de Artigas ante el Congreso; y más tarde pagaría su deslealtad con la vida.

El sector artiguista partía de la urgente necesidad de crear la existencia estadual de la provincia, de recrear el contexto necesario para ejercer el pleno derecho a través de sus órganos de Estado y decidir sobre las cuestiones de política local. Para esto, se debía lograr primeramente la organización política e institucional a fin de garantizar la preservación de los derechos autónomos.

Como podemos apreciar, eran dos interpretaciones de un momento histórico extremadamente complicado y riesgoso: el primer grupo daba primacía a intereses económicos y sociales, por lo que la propuesta era crear una estructura estadual acorde a esas pretensiones; en oposición, se encontraban aquéllos que consideraban como imprescindible para el crecimiento económico de estas provincias una plena autonomía territorial y autodeterminación política de los intereses porteños.

\footnotetext{
${ }^{2}$ Archivo General de la Provincia de Corrientes. Correspondencia oficial, Tomo 5, Folio 36.

${ }^{3}$ Wenceslao Néstor Domínguez, El primer Congreso correntino (Buenos Aires. 1964), pp. 47 y 48.
} 
Pero con el transcurrir del tiempo la coincidencia entre artiguismo y la doctrina correntina quedaría manifiesta en una serie de factores concurrentes; entre los cuales, particularmente la provincia de Corrientes incorporó aspectos geohistóricos que afianzarían su posición.

Coincidencias para enfrentar a Buenos Aires, la necesidad de frenar la potencialidad expansiva del Brasil, la prioridad de que el comercio exterior no lo maneje solo un puerto excluyendo a otros, la obsesión por un Gobierno republicano, son algunos de los postulados que presentaban en común. Todavía más, el artiguismo y la provincia de Corrientes fueron quienes enfrentaron militarmente a Buenos Aires de manera decidida y contundente.

Aquel nexo iría germinando a partir de las primeras manifestaciones de soberanía expresadas por el Cabildo de Corrientes, la adhesión a Artigas en 1814, y continuaría con los postulados más sobresalientes en la historia pactista interprovincial correntina, hasta alcanzar la impronta del Pacto Federal de 1831.

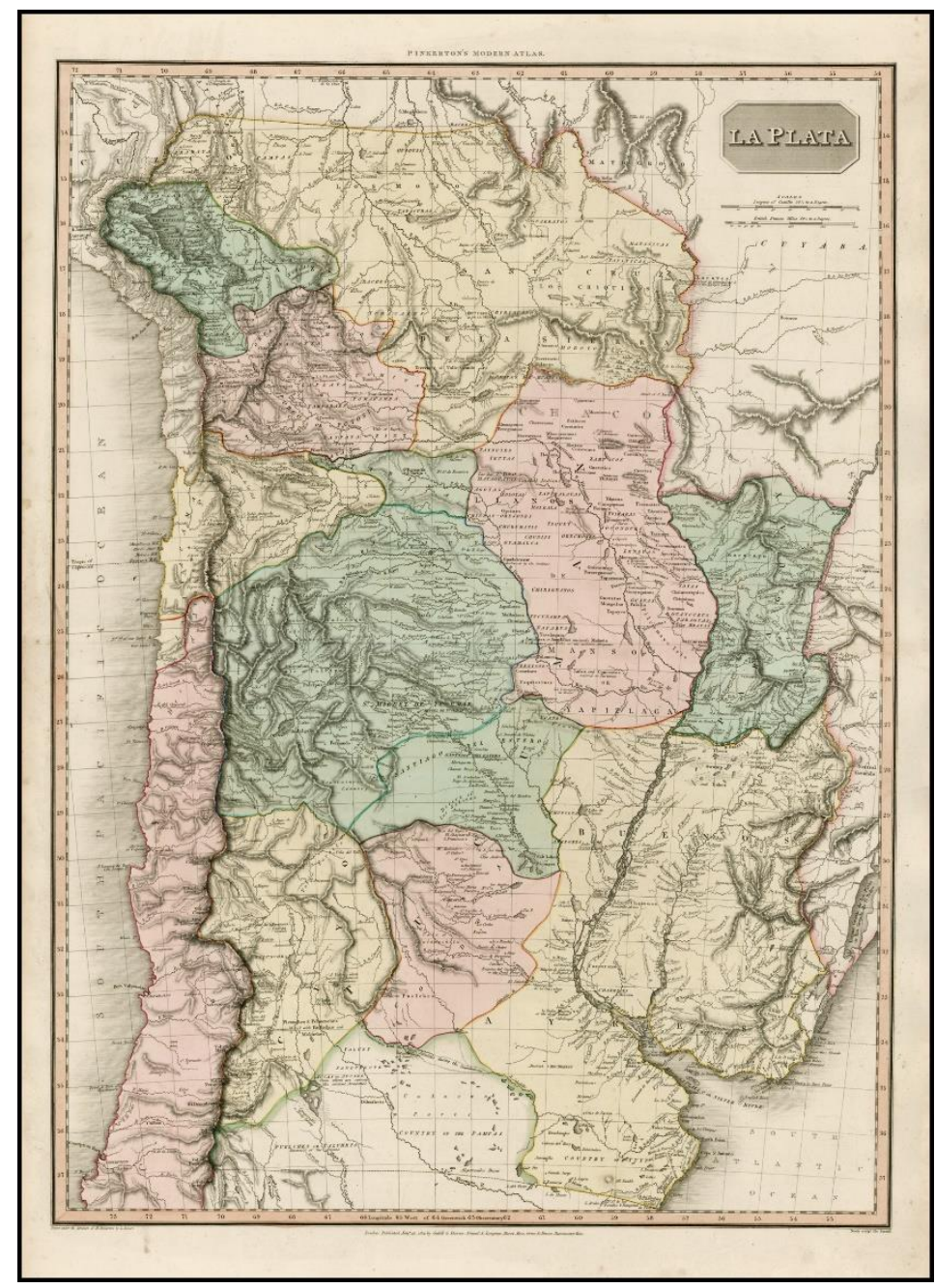

Mapa político de la región del Plata donde se observa la división administrativa del sistema de Intendencias en la década de 1810 a 1820. John Pinkerton, London 1818. Mapoteca del autor. 
Los principios básicos del artiguismo fueron instrumentados por Corrientes en su proyecto de Pacto de 1830, manteniendo de modo idéntico la actitud unificadora que implicaba la idea de federalismo, la autonomía de las instituciones provinciales y la política de habilitar puertos para el comercio exterior. ${ }^{4}$ El proyecto de Pedro Juan Ferré se convertía, de este modo, en la continuidad de los primeros principios artiguistas; no así, en cambio, el Pacto firmado el 4 de enero de 1831, en el que no se lograrían siquiera postulados básicos enclavados por el pensamiento de Artigas y, esta exclusión del federalismo económico del Pacto definitivo revelaría un claro triunfo de la diplomacia bonaerense.

Cabe decir que cuando Buenos Aires propuso el federalismo, éste resultó ficticio y parcializado. El Puerto no necesitaba del federalismo y ello se visualiza claramente en el sistema federal contemporáneo argentino. Los intereses sectoriales del Puerto mutaron en la segunda parte del siglo XIX, al instalarse con la figura del Estado Nacional para ir consolidándose definitivamente durante el siglo XX.

Aquel fortísimo vínculo entre artiguismo y provincia de Corrientes se manifestaría claramente en dos aristas referenciales, las que fueron fundamentales, tanto para uno como para otro: el federalismo y el republicanismo. A tal extremo llegamos que considero que la construcción del constitucionalismo correntino basado en un férreo republicanismo, como así también las sucesivas muestras de federalismo provincial, derivaron de la matriz propuesta por José Artigas -la que irrumpió claramente en 1814-; a partir de la cual había comenzado a despuntar una identidad local propia, característica y peculiar de las décadas preconstituyentes y traspasada a la construcción doctrinaria del pensamiento de los hombres de la provincia.

La provincia de Corrientes, en las primeras décadas del constitucionalismo rioplatense, bregó contra las mismas fuerzas que contrarrestaron al artiguismo, a saber, aquellas fuerzas centrípetas que la capital porteña venía ejerciendo desde los primeros momentos sobre el territorio de la antigua jurisdicción virreinal y que, posteriormente, tendrían identidad propia.

De manera que el derrotero artiguista contó, hasta último momento, con la colaboración correntina, tal cual lo manifiesta la firma del Pacto de Ábalos (24 de abril de 1820), donde se ratificaron los principios federales y la conformación de un Congreso organizativo. ${ }^{5}$ Sin embargo, estos episodios representaron los últimos intentos en tiempos de

\footnotetext{
${ }^{4}$ Dardo Ramírez Braschi, La provincia de Corrientes y el Pacto Federal de 1831. Disputas sobre federalismo y organización política en la etapa preconstituyente argentina (Corrientes: Moglia Ediciones, 2017).

${ }^{5}$ Federico Palma, "El Congreso de Ábalos", Ensayos, estudios y monografías, N. ${ }^{\circ} 5$ (1951), Universidad de la República (Uruguay), Instituto de Investigaciones Históricas.
} 
agonía del poder artiguista que estaba pronto a sucumbir, pues todos los caminos conducían a Artigas a territorio paraguayo.

Para concluir, creo necesario expresar que, entre las diversas cuestiones históricoculturales que unen a paraguayos y correntinos, tiene una valía preponderante la herencia de los valores del artiguismo, arraigados profundamente en las manifestaciones de autodeterminación y defensa de derechos locales, que durante el siglo decimonónico fue materia crucial y definitiva.

\section{Referencias bibliográficas}

Busaniche, José Luis. Estanislao López y el Federalismo del Litoral, Buenos Aires: Editorial Universitaria de Buenos Aires, 1969.

Caillets-Bois, Ricardo R. Rosas y la Comisión Representativa creada por el Pacto Federal de 4 de enero de 1831. Buenos Aires: Casa Jacobo Peuser Ltda., 1941.

Contribuidores UBA: Documentos relativos al Tratado del 4 de enero de 1831. Documentos para la historia argentina, Vol. 16. Facultad de Filosofía y Letras, Instituto de Historia Argentina "Doctor Emilio Ravignani". Buenos Aires: Editor Compañía SudAmericana de Billetes de Banco, 1922.

Demicheli, Alberto. Formación Constitucional Rioplatense, (Tres tomos). Buenos Aires: Roque Depalma Editor, 1956.

Domínguez, Wenceslao Néstor. El primer Congreso correntino, Buenos Aires: 1864.

Ferré, Pedro. Memorias del Brigadier General Pedro Ferré, octubre de 1821 a diciembre de 1842 (Contribución a la Historia de la provincia de Corrientes en sus Luchas por la Libertad y contra la Tiranía). Buenos Aires: Editorial Coni, 1921.

Gómez, Hernán F. El general Artigas y los hombres de Corrientes. Corrientes: Editorial Amerindia, 2010. . Historia de la provincia de Corrientes. Desde la Revolución de Mayo al Tratado del Cuadrilátero. Corrientes: Amerindia Ediciones Correntinas, 1997.

González Calderón, Juan A. Artigas Precursor del Federalismo Republicano (Las Instrucciones del Año XIII). Montevideo: Ed. Instituto Histórico y Geográfico del Uruguay, 1963.

Leyes de la Provincia de Corrientes (1821-1830) sancionadas por los Congresos Constituyentes Generales y Permanentes. Tomo I, Corrientes: Imprenta del Estado, 1929. 
Mantilla, Manuel Florencio. Crónica histórica de la Provincia de Corrientes. Tomo I, Buenos Aires: Banco de la provincia de Corrientes, 1972.

Miranda, Héctor. Las Instrucciones del Año XIII, (Dos tomos). Ministerio de Instrucción Pública y Previsión Social. Montevideo: Biblioteca Artigas, Colección de Clásicos Uruguayos (Vol.46), 1964.

Palma, Federico. "El Congreso de Ábalos", Ensayos, estudios y monografías, No 5 (1951), Universidad de la República (Uruguay), Instituto de Investigaciones Históricas.

Ramírez Braschi, Dardo. La provincia de Corrientes y el Pacto Federal de 1831. Disputas sobre federalismo y organización política en la etapa preconstituyente argentina. Corrientes: Moglia Ediciones, 2017.

Sagarna, Antonio. Los Pactos Preexistentes en la Constitución Nacional. Conferencia leída en: Universidad Nacional del Litoral (Santa Fe), el 24 de octubre de 1936. Buenos Aires, 1937.

\section{Cuando el Paraná fue un Rubicón.}

\section{Hechos, preguntas y ¿certezas? sobre José Artigas en Paraguay}

\section{Tomás Sansón Corbo*}

El 5 de setiembre de 1820 José Artigas cruza el Paraná e ingresa a Paraguay por Itapuá, la actual Encarnación. Quedaba atrás una década de lucha en la Banda Oriental. Una etapa jalonada por victorias y por derrotas. Tiempo de fragua de un pensamiento libertario, republicano y federal. Errores tácticos, flaquezas humanas y traiciones variopintas lo condujeron hasta Candelaria. ${ }^{6}$ Culminaba el frenesí revolucionario y comenzaba otra etapa. El Paraná fue su Rubicón, alea jacta est. Una nueva vida le esperaba en el territorio gobernado por José Gaspar Rodríguez de Francia.

\footnotetext{
${ }^{*}$ Universidad de la República (Uruguay). slbt@ hotmail.com

${ }^{6}$ La presencia de Artigas en Paraguay ha sido objeto del interés de intelectuales uruguayos y extranjeros desde la década de 1830. Las primeras noticias se conocieron gracias al testimonio de viajeros o funcionarios que por diversos motivos tuvieron contacto con él. Los textos referidos al tema son varios y no corresponde reseñarlos en este artículo pues, en general, se trata de versiones que abrevan en las mismas fuentes. De todos modos, hay tres obras que pueden considerarse canónicas y de consulta ineludible: Daniel Hammerly Dupuy, "Rasgos biográficos de Artigas en el Paraguay", en Artigas. Estudios publicados en "El País" como homenaje al jefe de los Orientales en el centenario de su muerte, 1850-1950, ccoordinado por Edmundo Narancio (Montevideo: Ediciones de El País, 1949); Nelson Caula, Artigas ñemoñaré I. Vida privada de José G. Artigas. Las ocho mujeres que amó. Sus catorce hijos. Develando su ostracismo y su descendencia en Paraguay (Montevideo: Rosebud Ediciones, 1999); Ana Ribeiro, El Caudillo y el Dictador (Montevideo: Planeta, 2003).
} 
¿La retirada se materializó en el marco de un "repliegue táctico”? ¿El caudillo oriental pretendía una alianza militar con el Dictador Supremo de la República? ¿Procuraba volver a la Banda Oriental con refuerzos para continuar la lucha? O, por el contrario, ¿tenía conciencia de su derrota? ¿La carencia de recursos humanos y económicos lo convenció de la imposibilidad de seguir combatiendo? ¿Por qué no retornó a "su patria”? ¿Razones de edad, deseo de no participar en luchas fratricidas, negativa del gobernante? Son preguntas para las que no hay respuestas certeras y sobre las que se debatió desde la propia creación del Estado Oriental.

El periplo de Artigas en Paraguay es conocido: unos meses de confinamiento en el convento asunceno de La Merced, veinticinco años de aislamiento en la villa de San Isidro de Curuguaty y, por último, un lustro de residencia en las afueras de la capital bajo la protección de Carlos Antonio López.

Daniel Hammerly Dupuy plantea —en un curioso intento de "mapeo psicológico"que, mientras estuvo en Paraguay, el ilustre extranjero:

[...] pasó por tres etapas de duración desigual. Primeramente, respondió a las reclamaciones lógicas de su mente que le sugería proyectos democráticos de vasto alcance político. Cuando la actitud del Dictador Perpetuo trabó su libertad política, entró en una nueva etapa psicológica, que reclamó después de las primeras reacciones un acomodamiento a las circunstancias, superando las dificultades mediante las tareas que contemplaban la satisfacción de la labor misma y el bienestar de los menesterosos. La etapa póstuma fue biológica y espiritual, su duración fue determinada por la vitalidad del organismo y templada por las esperanzas de orden trascendente. ${ }^{7}$

En Curuguaty, Artigas llevó una existencia apacible, alterada solo por la visita de Aimé Bonpland (1831). En Ibiray, por el contrario, tuvo una vida social más activa: recibió la visita de su hijo José María y de personalidades como Alfredo Demersay, José María Paz, Enrique Beaurepaire-Rohan y Rómulo Yegros.

Especialmente el carácter de las relaciones entre el doctor Francia y Artigas es uno de los asuntos que más curiosidad e interés ha generado entre los historiadores. Algunos autores

\footnotetext{
${ }^{7}$ Daniel Hammerly Dupuy, "Rasgos biográficos de Artigas" ..., p. 253.
} 
piensan que fue un prisionero. Otros, por el contrario, plantean que fue recibido como un asilado político.

En cualquier caso, el dictador le aseguró los recursos necesarios para que llevara una vida digna y, además, no accedió a la solicitud del "Supremo Entrerriano" (Francisco "Pancho" Ramírez) para que se lo entregara con el propósito de juzgarlo. Francia comentaría que recibió al caudillo porque consideró "un acto, no sólo de humanidad, sino aún honroso para la República el conceder un asilo a un jefe desgraciado que se entregaba". ${ }^{8}$ Desde esta perspectiva, el gobernante paraguayo podría ser considerado como precursor del Derecho de Asilo en América.

También brindó asistencia material y protección a varias decenas de personas que acompañaron a Artigas en su retirada. Se trataba de un grupo de "lanceros negros" que fueron ubicados en terrenos aptos para la agricultura, a dos leguas de Asunción. Formaron una comunidad de afrodescendientes, denominada Cambá Cuá, que sobrevive en la actualidad.

Después de todo, Artigas y Francia nunca se encontraron. El dictador sabía que el caudillo oriental le solicitaría apoyo para retornar al escenario de lucha y recuperar la autoridad perdida. Consideraba totalmente inconveniente una empresa de ese tipo, tanto por la situación interna de Paraguay como por el contexto geopolítico internacional (la "anarquía" imperante en las provincias del antiguo Protectorado artiguista, la Banda Oriental ocupada por Portugal...). Sus prioridades eran asegurar la independencia paraguaya y sostener una política de no intervención en los conflictos regionales.

Interpretaciones sobre las razones del ingreso de Artigas a Paraguay, reflexiones sobre su estatus jurídico, especulaciones en torno a la constitución de una familia junto a Clara Gómez Alonso (con quien habría tenido un hijo llamado Juan Simeón), ${ }^{9}$ motivos por los que

\footnotetext{
${ }^{8}$ Citado en Juan Stefanich, “Artigas, Francia y el Paraguay. El ostracismo del prócer oriental en tierra paraguaya y orígenes del derecho de asilo en América", en Artigas. Homenaje en el centenario de su muerte, coordinado por Simón Lucuix (Montevideo: Imprenta Nacional, 1952), p. 396.

${ }_{9}$ Es necesario explicitar que las noticias sobre una hipotética familia de Artigas en Paraguay fueron sistematizadas por Nelson Caula. Obra curiosa y polémica, elaborada en base a tradiciones orales y con escaso sustento documental, en la que se intenta demostrar que, en Curuguaty, Artigas "compartió su vida con una mujer, con la cual tuvo un hijo. Clara Gómez Alonso y Juan Simeón respectivamente. Sus `ñemoñaré actuales nos abrieron las puertas de sus casas y, por primera vez las de su memoria". Para "probar" la seriedad de uno de los informantes caracteriza: es "un joven estudiante de Derecho, cuyo rostro tiene un indisimulable aire artiguista, el uso de la barba lo asemeja bastante a Santiago, el hijo de Artigas y Melchora Cuenca" (Caula, Artigas ñemoñaré..., p. 277). Las conclusiones del autor deben ser relativizadas en función de criterios metodológicos: se basa, fundamentalmente, en tradiciones orales a las cuales asigna el valor de testimonios; maneja los datos como si fueran alegatos de testigos o protagonistas cuando, en realidad, son informaciones transmitidas por presuntos descendientes sobre hechos acaecidos un siglo y medio atrás; aunque hipotéticamente pudiera aceptarse la veracidad de la información, no pueden admitirse juicios tan tajantes como los formulados sin fuentes escritas que los convaliden.
} 
no volvió a Uruguay... Son cuestiones que seguirán en debate mientras no se encuentren nuevos documentos que brinden respuestas satisfactorias.

¿Qué nos queda de los treinta años de residencia del patriarca oriental en suelo guaraní? O mejor, ¿qué se puede rescatar al margen de los conocimientos tradicionales sobre el tema? Un capital patrimonial intangible de profunda significación geopolítica en la larga duración y un legado espiritual.

El exilio de Artigas es uno de los principales emergentes de los vínculos pluriseculares entre Uruguay y Paraguay. Estas relaciones tienen una tradición profunda: se remontan a comienzos del siglo XVII, cuando el asunceno Hernando Arias de Saavedra (Hernandarias) introdujo el ganado en la Banda Oriental, y se proyectan hasta el presente en el contexto del Mercosur. Estas relaciones fraternas se materializan, por ejemplo, en la casi centenaria "Escuela Artigas" (erigida en el predio donde el prócer pasó sus últimos años) y en el frenesí musical del candombe en la comunidad de Cambá Cuá.

Precisamente, los vínculos entre las naciones y los procesos de integración regional deben tener, para ser efectivos y exitosos, un sustento cultural profundo. El conocimiento mutuo, la conciencia de tradiciones y convicciones compartidas, favorecen el entendimiento entre los gobiernos y los pueblos.

Por tanto, las tres décadas de residencia del prócer oriental en Paraguay integran el acervo inmaterial que explica y fortalece el patrimonio relacional entre ambos países. Rescatar y preservar ese acervo es necesario para nuestros "estados-frontera" que pugnan en el marco de la Cuenca del Plata, desde el período revolucionario, por mantener su independencia y soberanía frente a sus vecinos con pretensiones tradicionalmente hegemónicas, Brasil y Argentina.

Los vínculos y los sentimientos de hermandad, cimentados en el humus del exilio de Artigas, resultan muy fuertes entre los "socios menores" del Mercosur. En 1913, por ejemplo, se organizó una "peregrinación" de jóvenes uruguayos a Paraguay. La delegación oriental era portadora de diversos mensajes de confraternidad enviados por intelectuales y políticos. Uno de los más significativos fue el del autor de Ariel, José Enrique Rodó:

[...] el Uruguay es el Paraguay atlántico; el Paraguay es el Uruguay de los trópicos. Si alguna vez se interpuso entre ellos el humo del combate, los signos materiales de ese infausto recuerdo fueron ya de retorno, para demostrar que el fraternal amor salió acrisolado de la lid cruenta, porque se acrecentó con la 
recíproca admiración del heroísmo, que los mostró, en aquella aciaga ocasión, más semejantes que nunca. ${ }^{10}$

En otro orden de cosas, durante su residencia en Paraguay Artigas llevó una vida tranquila. Alejado de las turbulencias políticas y militares, se dedicó a tareas de labranza y a cultivar su espiritualidad.

Uno de los testimonios más interesantes sobre este asunto lo suministró el Padre Fidel Maíz, quien recibió valiosa información de parte de su hermano Francisco Ignacio Maíz (cura párroco de la villa de San Isidro de Curuguaty en vida de Artigas). ${ }^{11}$ De acuerdo a estos aportes, Francia le hacía llegar mensualmente una onza de oro con la cual Artigas costeaba sus necesidades e incluso ayudaba a los pobres de la localidad. La importancia de este dato radica, no tanto por confirmar el título de "Padre de los pobres" — reconocimiento proveniente de la historiografía tradicional-, sino porque ilustra un sentimiento que hoy podríamos llamar humanitario, pero que, en aquel entonces, indudablemente, era identificado como caridad cristiana. Esta conducta por otra parte, no era nada novedosa en quien desde su juventud estaba acostumbrado a vivir sin ostentación y con los recursos indispensables. Es muy posible que la impronta franciscana (heredada de su formación escolar en Montevideo) se proyectara hasta los últimos años de su existencia a través de pautas que marcaban su vida cotidiana.

Su hijo José María lo visitó en Ibiray (1846) y permaneció tres meses junto a él. En una crónica titulada Emigración del General Artigas al Paraguay. Su vida y situación, comenta acerca de los vecinos de la zona:

[...] pobres que tanto quieren y veneran a mi padre, se reúnen con él para rezar el rosario, cuando el toque de oraciones de las campanas distantes llega hasta ellos de la Asunción, los vi todos los días en el mismo sitio. Mi padre hacía coro; los demás arrodillados en torno suyo, contestaban las oraciones, muchos de ellos, la mayor parte, en guaraní. Al concluir todos se retiraban a sus casas, después de

\footnotetext{
${ }^{10}$ Citado en: Adriano Irala y Santino Barbieri, Paraguay - Uruguay. Las fiestas de confraternidad celebradas en Asunción con motivo de la peregrinación uruguaya al solar de Artigas (Buenos Aires: Compañía Sudamericana de Billetes de Banco, 1913), p. 155.

${ }^{11}$ Información recogida por Juan Zorrilla de San Martín, La Epopeya de Artigas (Montevideo: Biblioteca Artigas de Clásicos Uruguayos, 1963), t. V, p. 104.
} 
saludar, uno a uno, con veneración al viejo, éste entraba a paso lento en su rancho, y se acostaba muy temprano. ${ }^{12}$

Como bien sabemos, el rosario era una de las devociones familiares más comunes en la América hispana desde los tiempos de la colonia. El caudillo, entonces, no hacía más que retomar una práctica piadosa que había aprendido en el seno de su hogar y que seguramente debió suspender en los ajetreados años de su vida pública. Se conocen otros testimonios relacionados con la asidua participación de Artigas en la misa dominical ${ }^{13}$ y sobre su voluntad de recibir los últimos sacramentos en el momento postrero de su existencia. ${ }^{14}$

Ahora bien, poco se puede avanzar para responder la guirnalda de interrogantes que hemos referido sobre las tres décadas de presencia de Artigas en Paraguay. Las fuentes son escasas y el historiador no tiene otra opción que especular en torno a las mismas, aunque sin forzar conclusiones.

¿Qué certezas nos quedan entonces? El propio fenómeno del ostracismo y sus derivas constituyen un reservorio de tópicos (ideológicos, simbólicos y diplomáticos) que ilustran y nutren el patrimonio cultural intangible que une a Paraguay y a Uruguay. Los indicios sobre las prácticas religiosas y devocionales del caudillo, en Curuguaty e Iviray, dan cuenta de su espiritualidad, dimensión totalmente soslayada por la historiografía oficial uruguaya hasta fines del siglo $\mathrm{XX} .^{15}$

En 1841, luego de la desaparición física del doctor Francia, los cónsules Carlos Antonio López y Mariano Roque Alonso comunicaron al anciano residente en Curuguaty que tenía plena libertad para volver a su patria. Aun así, Artigas prefirió quedarse, por lo que solicitó a los gobernantes licencia para permanecer allí hasta el término de sus días. Como a muchos otros viajeros, comenta Carlos Pastore: “...la naturaleza paraguaya había realizado el

\footnotetext{
${ }^{12}$ Testimonio de José María Artigas citado en Zorrilla de San Martín, La Epopeya, t. V, pp. 294-295.

${ }^{13} \mathrm{Al}$ respecto, nos parece relevante lo que consigna Ana María Ribeiro: "Son pocos los testimonios orales que pudieron recogerse en esa aldea [Curuguaty] que, en la década siguiente a su muerte, durante la guerra de la Triple Alianza, fue totalmente quemada y destruida. Algunos de sus habitantes regresaron después de la masacre, y entre ellos (en 1926), Plácido Jara buscó recuerdos. Habló con dos ancianos de la Villa, un hombre de ochenta años y una mujer de cien, que recordaban haber oído a sus mayores: 'haber llegado a Curuguaty enviado por el Superior Gobierno un ilustre expatriado y extranjero a quien se rendía respeto'; agregando que 'un señor extranjero, más moreno que blanco, vistiendo un largo saco asistía a oír misa todos los domingos a la iglesia' (...)", en: Los tiempos de Artigas (Montevideo: Ediciones de El País, 1999), t. VI, p. 77.

${ }^{14}$ La descripción de la celebración le fue suministrada a Juan Zorrilla de San Martín por Monseñor Bogarín, obispo de Asunción, quien había sido informado por una dama que participó de la misma (Cf. Zorrilla de San Martín, La Epopeya, t. V, p. 321).

${ }^{15}$ Cfr.Tomás Sansón Corbo, "La religiosidad de Artigas", en Nuevas miradas en torno al artiguismo, coordinado por Ana Frega y Ariadna Islas (Montevideo: Facultad de Humanidades y Ciencias de la Educación, 2001); y Pedro Gaudiano, Artigas católico (Montevideo: Universidad Católica, 2002).
} 
milagro de aprisionarlo con su belleza y el pueblo de retenerlo con sus virtudes hospitalarias". 16

El 23 de setiembre de 1950, en Ibiray, luego de recibir los últimos sacramentos administrados por el Presbítero Cornelio Contreras, fallecía el prócer oriental y cumplía así su voluntad de morir en Paraguay.

\section{Referencias bibliográficas}

Caula, Nelson. Artigas ñemoñaré I. Vida privada de José G. Artigas. Las ocho mujeres que amó. Sus catorce hijos. Develando su ostracismo y su descendencia en Paraguay. Montevideo: Rosebud Ediciones, 1999.

Gaudiano, Pedro. Artigas católico. Montevideo: Universidad Católica, 2002.

Hammerly Dupuy, Daniel. "Rasgos biográficos de Artigas en el Paraguay”. En Artigas. Estudios publicados en "El País" como homenaje al Jefe de los Orientales en el centenario de su muerte, 1850-1950. Coordinado por Edmundo Narancio. Montevideo: Ediciones de El País, 1949.

Irala, Adriano y Barbieri, Santino. Paraguay - Uruguay. Las fiestas de confraternidad celebradas en Asunción con motivo de la peregrinación uruguaya al solar de Artigas. Buenos Aires: Compañía Sudamericana de Billetes de Banco, 1913.

Pastore, Carlos. “Artigas en el Paraguay”. En Artigas. Homenaje en el centenario de su mиerte. Coordinado por Simón Lucuix. Montevideo: Imprenta Nacional, 1952.

Ribeiro, Ana. Los tiempos de Artigas, t. VI. Montevideo: Ediciones de El País, 1999. El Caudillo y el Dictador. Montevideo: Planeta, 2003.

Sansón Corbo, Tomás. "La religiosidad de Artigas". En Nuevas miradas en torno al artiguismo. Coordinado por Ana Frega y Ariadna Islas. Montevideo: Facultad de Humanidades y Ciencias de la Educación, 2001.

Stefanich, Juan. “Artigas, Francia y el Paraguay. El ostracismo del prócer oriental en tierra paraguaya y orígenes del derecho de asilo en América". En Artigas. Homenaje en el centenario de su muerte. Coordinado por Simón Lucuix. Montevideo: Imprenta Nacional, 1952.

Zorrilla de San Martín, Juan. La Epopeya de Artigas, t. V. Montevideo: Biblioteca Artigas de Clásicos Uruguayos, 1963.

\footnotetext{
${ }^{16}$ Carlos Pastore, “Artigas en el Paraguay”, en Artigas. Homenaje en el centenario de su muerte, coordinado por Simón Lucuix (Montevideo: Imprenta Nacional, 1952), p. 25.
} 


\section{Una institución educativa fundamental: la Academia Literaria}

\section{David Velázquez Seiferheld*}

En 1842 comenzó su actividad educativa la Academia Literaria, creada por los cónsules Mariano Roque Alonzo y Carlos Antonio López el 30 de noviembre de 1841. A su vez, daba cumplimiento a una de las resoluciones más importantes del Congreso reunido el 12 y 13 de marzo de ese mismo año. ${ }^{17}$

Del decreto de creación extraemos los fundamentos por los cuales fue constituida, así como sus fines fundamentales y las necesidades que pretendía satisfacer. ${ }^{18}$ En primer lugar, se iniciaba señalando que una misión fundamental de la autoridad era atender a las carencias graves de "ilustración pública" con miras a "restablecer los elementos de la ilustración enteramente extinguidos", prosigue el documento, en una abierta crítica a la dictadura de Francia en el aspecto educativo; como a la "grande escasez del clero nacional" y a la falta de "capacidades civiles" para la conducción del Estado y sus instituciones.

Por lo demás, el decreto contemplaba también la organización disciplinar: una cátedra de Latinidad, otra de Idioma Castellano y Bellas Letras. Las siguientes eran la cátedra de Filosofía racional "en método didáctico", la Teología dogmática "en igual método"; Historia Sagrada y cronología, e Historia eclesiástica y Oratoria sagrada. El método didáctico comprendía el conocimiento de las disciplinas de "lógica, metafísica, ética general y particular, física general y particular”. Además, se previó una conferencia semanal de elementos de religión cristiana para los alumnos desde la cátedra de Latinidad; y otra sobre los Derechos y Deberes del Hombre Social desde la cátedra de Idioma Castellano.

Por entonces, se nombró director interino al presbítero Marco Antonio Maíz (¿1785? 1848), quien además fue designado catedrático de Latinidad; mientras que la cátedra de Idioma Castellano, y por ende las mencionadas conferencias, quedaron a cargo del presbítero

\footnotetext{
*Universidad Nacional de Villarrica del Espíritu Santo (UNVES). david.velazquez@unves.edu.py

17 Congreso Nacional de la República del Paraguay. Actas de las sesiones de los Congresos de la República, desde el año 1811 hasta la terminación de la Guerra. Asunción: Tipografía del Congreso, 1908. pp. 32-33.

${ }^{18}$ Decreto de Fundación de la Academia Literaria. En ANA - SH - 245n22-223-262.
} 
José Joaquín Palacios. Este sacerdote argentino había llegado al Paraguay presumiblemente hacia 1840; actuó como consejero de Carlos Antonio López, primero como cónsul y luego como presidente de la República. Según Scavone Yegros, sería quien en 1842 habría de proponer al Congreso la ratificación de la independencia. Años después, enemistado con López, volvería a su país. ${ }^{19}$

En tanto proyecto de formación de una élite republicana, la creación de la Academia era la base de un programa de mayor alcance que, sin embargo, no llegó a concretarse: "ha de servir de plantel para el colegio que se ha de establecer en esta capital".

Por decreto del 27 de enero de 1842, el consulado resolvió que para el funcionamiento de la Academia fueran entregados "los fondos del Seminario conciliar suprimido en el régimen pasado", es decir, los del Seminario de San Carlos (el que en tiempos del Paraguay provincial fuera colegio real). Además, en cumplimiento del mandato del Congreso de 1841, también se otorgaron los salarios no cobrados por el dictador Francia para el financiamiento de la nueva institución educativa.

En este mismo decreto se daba a conocer el reglamento general de la Academia, con disposiciones relativas a los horarios, los programas, las normas de comportamiento de los alumnos y de los catedráticos, etc. Entre otros puntos, se establecía que el año escolar comenzaba "en ceniza" y concluía el día de Navidad. Luego, era tiempo de vacaciones. Las clases tenían lugar de lunes a sábado al mediodía, así como los días festivos de precepto. Los exámenes eran públicos y los examinadores eran designados no por las autoridades de la Academia, sino por "el Supremo Gobierno". En el receso, los alumnos llevaban tareas a sus casas.

En cuanto a las normas de comportamiento y disciplinarias, el reglamento asignaba a los padres el compromiso de "mandar a sus hijos a la academia a las horas designadas, lavados, peinados y aseados", y que "los hagan estudiar en sus casas, y comportarse en todo". El director, por su parte, debía prohibir a los alumnos que "estén en grupos en las clases, ni en chacotas, ni usen de gritos ni de otras acciones indecentes". La expulsión de los alumnos podía darse por faltas injustificadas reiteradas, así como por un número "considerable" de lecciones desaprobadas, y por ser "incorregibles en defectos graves". Además, estaba

\footnotetext{
${ }^{19}$ Manuel Pedro de Peña es uno de los que más detalles brinda sobre las actividades de José Joaquín Palacios en el Paraguay, en las polémicas cartas que públicamente dirigió a Francisco Solano López en 1865. Probablemente, exagera la influencia del sacerdote argentino en el afán de menoscabar la figura de Carlos Antonio López. En González de Bosio, Beatriz, La legión paraguaya. Asunción, 2014. pp. 103-105. En línea en: http://www.corredordelasideas.org/docs/ppt/legion_paraguaya.pdf. Véase, además: Scavone Yegros, Ricardo, La declaración de la independencia del Paraguay (Asunción: Servilibro, 2011), p. 141.
} 
prohibido tanto a padres como a encargados que retirasen a los estudiantes en horas de clases. Se excluía el castigo corporal y se admitía solo el "puramente penitenciario".

Las reglas que los alumnos debían memorizar eran las siguientes: hablar con la verdad; presentarse "aseados y limpios en todo el cuerpo y en la ropa"; mantener silencio durante las clases y las tareas; ser bondadoso con los hombres "y también con los animales", "huir de toda mala compañía", no pronunciar groserías ni inventar apodos; "no hacer burla a personas feas, cojas, o de otro modo defectuosas", "estudiar para aprender y ser útiles a sus semejantes". Además, debían "ser obedientes a sus padres y superiores y obsecuentes a sus mayores", evitar siempre las peleas y "también los debates sobre asuntos impertinentes"; y "adorar a Dios como a primera causa y obedecer a superiores y maestros". ${ }^{20}$ Una vez a la semana, cada catedrático realizaba un examen de estas pautas. Entre otras restricciones, los alumnos no podían reunirse en la entrada del local de la Academia; y fuera de la institución también debían mantener cierto comportamiento: les estaba prohibido jugar en carnaval, concurrir a riñas de gallos, asistir a las tabernas, jugar a las barajas y "envites" y manipular cuchillos.

Por otro lado, para ingresar a la Academia literaria como alumno externo, el candidato debía dar sus datos de filiación y procedencia y demostrar "saber leer y escribir correctamente; tener si es posible conocimiento de las primeras reglas de la aritmética". En cuanto a la dinámica escolar, ella era regulada mediante el uso de la campana que marcaba los tiempos de gimnasia, clases en aulas, recreos y conferencias.

Ateniéndonos a la orden de exámenes realizados el 19, 20, 21 y 22 de diciembre de $1843,{ }^{21}$ observamos que el programa estaba organizado de la siguiente manera: la Primera Clase, de conocimientos introductorios, estaba dividida en seis fracciones, de contenidos de complejidad creciente (homólogas de los grados actuales); mientras que la segunda clase consistía en conocimientos más complejos y avanzados, divididos en dos fracciones también organizadas por niveles de complejidad. A diferencia de la moderna organización en grados, los alumnos no estaban distribuidos por edades. Los exámenes eran públicos; y cabe agregar que una nota institucional destacaba que algunos alumnos no habían podido completar el año lectivo ya que debieron abandonar la Academia al ser víctimas de la viruela.

\footnotetext{
${ }^{20}$ Estas mismas reglas aparecen en un Reglamento de Escuelas de Capiatá, en 1864. Véase David Velázquez Seiferheld, "Hacer aprender a los niños de memoria todos los artículos concernientes a ellos...Un reglamento de escuela de primeras letras de tiempos de Francisco Solano López”. En Ana Paula Squinelo e Ignacio Telesca, (organizadores), 150 anos após - A guerra do Paraguai: entreolhares do Brasil, Paraguai, Argentina e Uruguai, Volumen 3. Grupo de Pesquisa Historiografía e Ensino da História - Universidad Federal de Mato Grosso Do Sul, Campo Grande/Ms, 2019, pp. 435-458.

21 "Programa de exámenes de la Academia Literaria". ANA - SH - 267n11.
} 
¿Cuáles eran los contenidos que debían acreditar los alumnos de la Academia? En Latín y Gramática, en "Del idioma hispano americano", "De la ortografía simplemente considerada", "De la Analogía y sus partes", "De la sintaxis", "De la proposición, frase o periodo", "De la construcción de las proposiciones", "Diversas clases de proposiciones", "De las sintaxis figuradas"; "Conocimientos ortográficos sobre la pronunciación, uso constante y origen de las voces", "Observación sobre las abreviaturas", "Breve relación de números romanos". En Bellas Letras, los contenidos que aparecen son: "Elocuencia y demás géneros en prosa" -tema que engloba 31 capítulos, y que abarca los géneros literarios-, luego aparece "Poesía", de 17 capítulos. En esas páginas eran estudiados con detalle "La Ilíada y la Odisea de Homero", "La Eneida de Virgilio", "Farsalia” de Lucano y “Jerusalén”, de Tasso.

Seguidamente, corresponde el estudio del "Tratado de los Deberes y Derechos del Hombre Social" y los "Aditamentos para la Instrucción del Hombre Social". Aparecen a continuación, los llamados Estudios Accesorios: "Del conocimiento de Dios por sus obras", que presentaba articulación con las enseñanzas de primer año: contenido organizado en 17 Diálogos que versaban sobre la creación y la naturaleza. Este segmento reviste gran interés porque luego de un desarrollo en torno a los animales y plantas, así como a los elementos, en el último diálogo se abordaba el tema "De las producciones de diversos países y su uso en el comercio".

Los saberes reunidos en el eje "Varias sentencias, máximas morales y pensamientos. Industria y Vigilancia" incluían: "Medidas y pesas", "Medidas de extensión y del tiempo", "Cronología en general y algunas aplicaciones particulares", "Modos de formar las efemérides: también las nacionales", "Varios poemas heroicos y sublimes, Letrillas, Epigramas, Sonetos y Églogas”.

Puntualmente, el 9 de febrero de 1842 tuvo lugar la apertura de clases, con 149 alumnos, 23 de ellos internos. Entre los estudiantes aparecen apellidos guaraníes: Juan Andrés Cairay, Blas Andrés Yaguareté, Bartolomé Paraná, Juan Pedro Maningá; Juan Francisco Taicó, Pedro Manuel Yabaité. El director interino de la Academia, Presbítero Marco Antonio Maíz pronunció la alocución inaugural en la que definía la jornada como el día "en que se han fijado los fundamentos de la felicidad paraguaya". Además, exhortaba a los padres al "gozo recompensador" de sus esfuerzos al "ver a vuestros hijos fundadores de este plantel literario en donde bien instruidos en los principios elementales ya de la Religión ya de los derechos del hombre social y de las otras ciencias (...) serán con el tiempo los miembros útiles de la Patria". Para aquellos, serán "apoyos consoladores" y para la sociedad, "transmitiendo este ejemplo de gente en gente, contribuirán directamente a la elevación de este País de su 
nacimiento". Con igual entusiasmo, animaba a los estudiantes: "vuestra garantía de progreso es vuestro honor, vuestra virtud, vuestra aplicación y obediencia". ${ }^{2}$

En 1843, el gobierno decretó la creación de una cátedra de Filosofía dentro de la Academia Literaria, al mismo tiempo que definió una política de gratuidad para que los alumnos destacados de primeras letras de la campaña pudieran concurrir a la institución, evitando pretextos, y, además, ante la "indolencia" de los padres por la educación de sus hijos $^{23}$.

Por decreto del 28 de octubre de 1843 el consulado ordenó a los jueces comisionados que se dispusieran "todas las medidas, prudentes y eficaces" con las familias, para que éstas pudieran enviar a sus hijos a cursar estudios. La norma establecía que los comisionados debían convocar a los vecinos, así como a los maestros de primeras letras de sus jurisdicciones con el propósito de dar a conocer la normativa, y que estos últimos informaran sobre "los jóvenes capaces de estudios mayores". ${ }^{24}$ Los jueces, por su parte, anunciaron prontamente a la población sobre el decreto, la cual manifestó "gozo y contento" en todas las ocasiones. En algún caso, como en Yataity, incluso el juez ordenó que se comunicaran las disposiciones "explicándolas en idioma guaraní".

Luego, jueces y maestros se debían abocar a la tarea de identificar a los "jóvenes capaces", para proporcionar las listas de sus respectivas jurisdicciones. Las edades oscilaban, en promedio, entre los 12 y los 18 años. En algunos documentos, sin embargo, se advierte que había quienes que no podían enviar a sus hijos "por las escasas facultades" (en alusión a los padres), o bien, los jueces por sí mismos constataban la "falta de inteligentes" a pesar de lo que los maestros declaraban. Otros padres se manifestaban "ansiosos de darles a sus hijos esta educación; pero que se veían en estado de no poder asistir a sus hijos por el estado de suma pobreza en que cada uno se hallaba" por ser "sumamente insolventes". Entre otras razones, se explicaba que "no podían sufragarles los libros competentes al efecto", "no tenían como comprar para la decencia de sus hijos" (vestimenta y calzado) ... En algún caso, se comprometían a enviarlos al año siguiente, con más recursos o mejor formados. El juez comisionado de Luque, por ejemplo, informaba que la falta de "progreso" en las escuelas de campaña se debía a "la escasez de papel blanco y libros que de ordinario los padres pobres no pueden proporcionar" a los jóvenes.

\footnotetext{
22 "Discurso pronunciado en la inauguración de la Academia Literaria". ANA - SH - 254n13.

23 "Creación de la cátedra de Filosofía en la Academia Literaria”. ANA - SH - 257n1.

24 “Circular sobre la creación de la cátedra de Filosofía en la Academia Literaria y orden a los comisionados de gobierno para que exhorten a los padres de familia a enviar a sus hijos a recibir la enseñanza gratuita”. ANA $\mathrm{SH}-256 \mathrm{n} 21$.
} 
La Academia literaria cumplió un papel fundamental respecto de los fines para los cuales fue creada. Constituyó el espacio en el que se formó el clero, la magistratura o la burocracia estatal, y, además, buena parte de los egresados de la Academia literaria se convertirían en maestros de primeras letras en distintos partidos. Estaban imbuidos, ciertamente, de la pedagogía tradicional de la lección magistral, el dictado y la palmeta; pero también de contenidos más amplios y mejor formación que sus precedentes. En su mensaje de 1844, el presidente Carlos Antonio López declaraba al Congreso: "La construcción del colegio está pendiente (...) Entretanto se ha observado que la casa destinada para la academia suple bien a todos los servicios de un colegio." 25 Por ello, ya no haría falta crear el Colegio: la academia literaria cumplió esta importante función.

En particular, el aula de Filosofía daría, con el tiempo, origen a un movimiento intelectual importante, una de cuyas expresiones claves llegó a ser la revista La Aurora, publicada entre 1860 y 1861 con la dirección del publicista y docente español Ildefonso Antonio Bermejo (1820 - 1892).

Buena parte de la burocracia del Estado de entonces se formó con la impronta de aquellas premisas que permitían, además, una inédita e incipiente movilidad social a partir de las escuelas de primeras letras.

También el clero se revitalizó con sacerdotes formados en la Academia Literaria, lo que -a juzgar por el papel que concedía López a la Iglesia católica y el rol que cumplían la Religión y "el culto público" en la primera república- denota un logro nada desdeñable. En ocasión del reinicio de las relaciones con el papado, los cónsules mencionaron a Gregorio XVI, en nota dirigida a inicios de 1842, que se hallaban abocados a la formación del clero: no ciertamente a través del expediente de reabrir el antiguo Colegio Seminario, sino mediante la nueva Academia Literaria. Estos mismos sacerdotes fueron luego, en 1859, los catedráticos de la nueva instancia formadora del clero: el Seminario conciliar que comenzó sus actividades el 1 de abril.

Su importancia quedó además en evidencia con la denominación en 1849 de una calle: Academia Literaria, "la que sube de la cuadra de este establecimiento", la actual calle Montevideo de Asunción. ${ }^{26}$

Por último, es interesante observar que un documento procedente también de la Academia, serviría de base al pensamiento republicano de los primeros años de la

\footnotetext{
${ }^{25}$ Citado en Juan F. Pérez Acosta, Carlos Antonio López, obrero máximo (Asunción: Servilibro, 2011), pp. 462465.

26 "Decreto sobre denominaciones de calles". ANA - SH - 286n6.
} 
posdictadura de Francia: el "Tratado de los Derechos y Deberes del Hombre Social", de agosto de $1843 .{ }^{27}$ Por tratarse del texto de la cátedra de Idioma Castellano (y de las conferencias que el catedrático de la misma debía dictar semanalmente bajo el mismo título), su paternidad se debate entre José Joaquín Palacios (primer encargado de esta cátedra) y Carlos Antonio López, por entonces cónsul. La mayor parte del consenso gira en torno a este último.

Se trata de un documento en que las ideas de Montesquieu y el republicanismo clásico conviven con los ideales de virtud cristiana como modelo de ciudadano, tal como ocurrió en casi todas las repúblicas americanas durante décadas. Un republicanismo que se atenuaría, progresivamente, hasta casi pasar desapercibido bajo las formas crecientes de autoritarismo y cesarismo que habrían de caracterizar al estado paraguayo de los tiempos de los López.

\section{Referencias Bibliográficas}

González De Bosio, Beatriz. La legión paraguaya. Asunción, 2014, pp. 103-105. [http://www.corredordelasideas.org/docs/ppt/legion paraguaya.pdf]

Pérez Acosta, Juan F. Carlos Antonio López, obrero máximo. Asunción: Servilibro, 2011.

Scavone Yegros, Ricardo. La declaración de la independencia del Paraguay. Asunción: Servilibro, 2011.

Velázquez Seiferheld, David. "Hacer aprender a los niños de memoria todos los artículos concernientes a ellos... Un reglamento de escuela de primeras letras de tiempos de Francisco Solano López”. En Squinelo, Ana Paula y Telesca, Ignacio (organizadores), 150 anos após - A guerra do Paraguai: entreolhares do Brasil, Paraguai, Argentina e Uruguai, Volumen 3. Grupo de Pesquisa Historiografía e Ensino da História Universidad Federal de Mato Grosso Do Sul, Campo Grande/Ms: Editora Life, 2019.

\section{Documentos consultados:}

-Actas de las sesiones de los Congresos de la República, desde el año 1811 hasta la terminación de la Guerra. Asunción: Tipografía del Congreso, 1908.

\section{De la Sección Historia del Archivo Nacional de Asunción (SH- ANA).}

\footnotetext{
27 “Tratado de los deberes y derechos del hombre social”. ANA - SH - 258n4.
} 
"Circular sobre creación de la Cátedra de Filosofía en la Academia Literaria y orden a los Comisionados de Gobierno para que exhorten a los padres de familia a enviar a sus hijos a recibir la enseñanza gratuita”. PY - ANA - SH - 256n21-221-228.

“Decreto de Fundación de la Academia Literaria”. PY - ANA - SH - 245n22-223-262.

“Decreto sobre denominaciones de Calles”. PY - ANA - SH - 286n6-19-20.

"Discurso pronunciado en la inauguración de la Academia Literaria". ANA - SH - 254n13.

"Listas enviadas por varias escuelas del interior de la República, con nombres de Alumnos capacitados para estudios superiores en la Academia Literaria". PY - ANA - SH 258n6-87-119.

“Programa de exámenes de la Academia Literaria”. PY - ANA - SH - 267n11-52-61.

"Prospecto de una Academia Literaria a establecerse en Asunción”. PY-ANA-SH-251n6.1148-152.

"Tratado de los deberes y derechos del hombre social". ANA - SH - 258n4.

\section{Dicho y no dicho.}

\section{El encuentro entre Alfred Demersay y José Artigas en el Paraguay}

\section{Liliana M. Brezzo*}

En Paraguay, en torno al año 1847, José Artigas fue retratado por primera y única vez. Las circunstancias son conocidas. Luego de su definitiva derrota a manos de sus antiguos aliados de la Liga Federal, Estanislao López y Francisco Ramírez, en 1820, el caudillo oriental solicitó asilo a José Gaspar Rodríguez de Francia, el cual le fue otorgado con serias restricciones. De hecho, vivió prácticamente aislado en la villa de Curuguaty dedicado a labores agrícolas. A la muerte de Francia, en 1840, pasó a residir en Asunción y allí falleció en 1850, a los 86 años. En la investigación que dedica a "deconstruir" los retratos de Artigas, Laura Malosetti anoticia que el naturalista francés Alfred Demersay (1815 -1891) halló a Artigas en la capital del Paraguay y fue quien produjo el retrato que es considerado la única imagen "verdadera" del prócer uruguayo. Massimo Leone añade, en un texto reciente, que el origen del encuentro estuvo en el llamado del presidente López a Demersay para que tratase la

\footnotetext{
* CONICET, IDEHESI, Nodo Instituto de Historia, UCA. lilianabrezzo@ conicet.gov.ar
} 
enfermedad de su hijo (sin especificar a cuál de los cinco hijos se refiere) y que fue en esa temporada cuando dibujó el retrato de Artigas, que luego fue grabado por el francés Claude Sauvageot.

Surgen de estas escuetas referencias interrogantes que no han sido aun develados por completo ¿Qué tipo de relación estableció Demersay con Artigas durante su estancia en Paraguay? ¿Por qué Artigas accedió a posar para el pintor? ¿Con qué fines? ¿Por qué Demersay decidió publicar el retrato en el Atlas que editó años después en Paris? Al hilo de estas preguntas quisiera abundar, en esta breve intervención, en algunas de las observaciones proporcionadas por Leone y Malosetti para luego, a la luz de documentación proveniente del Archivo Nacional de Asunción, añadir algunos datos en torno al encuentro en Ibiray y sobre su incidencia en la representación del Paraguay que Demersay dio a conocer en Europa veinte años después, con motivo de la publicación de la Histoire physique, économique et politique du Paraguay.

Comencemos por repasar el hecho, ya profusamente documentado, de que la administración del presidente Carlos Antonio López representó, en el plano internacional, una modificación del enclaustramiento francista; procuró atraer el reconocimiento de la independencia del Paraguay y promovió la incorporación de tecnología e inmigración para el desarrollo económico del país. Uno de los acontecimientos acaecidos durante el primer año de su mandato fue decisivo en el proceso de construcción nacional: la introducción de la primera imprenta civil. De este modo, desde 1845 comenzaba a editarse el primer periódico en la historia paraguaya, El Paraguayo Independiente y, al año siguiente, apareció El Semanario. En ese empuje progresista hay que insertar el apoyo que López prestó a expediciones científicas patrocinadas por gobiernos de Europa y de Estados Unidos. De modo que el Paraguay resurgió como área de interés de conocimiento y exploración. ${ }^{28}$ Es sugestivo que, a poco de su designación para la Presidencia de la República, un naturalista sueco haya sido testigo de la transformación que se operaba: Eberhard Munck af Rosenschöld (1811-1868). Afincado en el Paraguay, se casó con Marcelina Rivarola y recogió sus impresiones en forma de cuatro cartas que fueron traducidas al portugués, francés y español. De ellas recibimos la primera impresión del gobierno de Carlos Antonio López visto por un extranjero: “López nunca salió de su país; no tuvo la menor parte en los negocios públicos: no pudo conocer las excelentes obras publicadas sobre los diversos ramos de la administración pública y economía

\footnotetext{
${ }^{28}$ María Victoria Baratta, "Paraguay en las crónicas de los viajeros durante el gobierno de José Gaspar Rodríguez de Francia” en Revista Paraguay desde las Ciencias Sociales, revista del Grupo de Estudios Sociales sobre Paraguay, nº 9 (2018): pp. 23-43.
} 
política ni tener la menor noticia de los acontecimientos de Europa y América en los últimos veinte años...Vivió retirado en el campo donde meditó sobre la situación y necesidades de su país". ${ }^{29}$ Sin perjuicio del testimonio del científico sueco, todo indica que Alfred Demersay fue el primer viajero proveniente de Europa que ingresó al Paraguay durante la administración de López. En el Archivo Nacional de Asunción figura la documentación que rodeó a las gestiones encabezadas por Francis La Porte, conde de Castelnau, fechadas en Fuerte Olimpo en febrero de 1845 para que la expedición científica francesa pudiera ingresar y recorrer el país. Figuran también dos notas extensas de Demersay dirigidas al presidente López: la primera, fechada en setiembre de 1845, en la que le presenta un breve bosquejo del estado de sus colecciones de historia natural; en tanto en la segunda nota, del 25 de febrero de 1846, le manifiesta su deseo de permanecer "todo el tiempo necesario" en Asunción para alcanzar el objeto de la misión geográfica y de historia natural que le fuera confiada. ${ }^{30}$ Esto nos indicaría que al momento del encuentro con Artigas, el científico francés ya había visitado al naturalista Aimé Bonpland, quien en ese entonces residía en Santa Ana. Fue, entonces, en este contexto en el que se encontraron Demersay y Artigas en Ibiray.

La realización de un retrato pictórico puede llevar, según sea el caso, entre horas o hasta el momento (quizás años) que se determine que está listo. El de Artigas parece haber sido realizado en un tiempo breve, tal vez, en el transcurso de una mañana o de una tarde. En el ya mencionado trabajo, Laura Malosetti sostiene que Demersay podría haberse servido de alguna lente o de la proyección de la sombra del modelo con técnicas como las que se usaban para hacer fisionotrazos. Este era un instrumento óptico mecánico, operado manualmente, inventado en 1786 por Gilles-Louis Chrétien, que se utilizó a lo largo del siglo diecinueve como una máquina de dibujo capaz de trazar los perfiles de objetos y modelos sobre láminas de cobre. Pero, lo que verdaderamente importa subrayar aquí, es que Demersay pudo observar el cuerpo y la cara de Artigas, y realmente los dibujó: el perfil aguileño y su mirada, sintetizada en un solo ojo, que para algunos especialistas "guarda algo del furor revolucionario" es la vera effigie, la verdadera imagen del rostro del prócer. ${ }^{31}$ El naturalista

\footnotetext{
${ }^{29}$ La edición portuguesa de las cartas, O Paraguay, seu pasado, presente y futuro por un estrangeiro que residió seis annosnaquellepaiz, fue publicada en 1848, con los auspicios de la legación del Paraguay en la Corte de Brasil, y obedeció a la necesidad de crear una imagen favorable del país cuando el ministro en la Corte imperial, Juan Andrés Gelly (1792 - 1859), negociaba los tratados de alianza y límites con Saturnino de Souza e Oliveira. En el mismo año, se publicó en Río de Janeiro una edición en francés y, al año siguiente, el escrito fue traducido al castellano y publicado en Asunción en la imprenta del Estado.

${ }^{30}$ República del Paraguay, Archivo Nacional de Asunción, Colección Historia de la República del Paraguay (ex Colección Río Branco), Py 443-1-12.

${ }^{31}$ Laura Malosetti Costa, "El primer retrato de Artigas: un modelo para deconstruir" en Caiana. Revista de Historia del Arte y Cultura Visual del Centro Argentino de Investigadores de Arte (CAIA), No 3 (2013): pp. 114.
} 
francés consignaría en la Histoire physique, économique et politique du Paraguay et des établissements jésuites, que diera a conocer en Paris entre 1860 y $1864,{ }^{32}$ que el retrato fue tomado del natural, es decir, teniendo el modelo ante la vista.

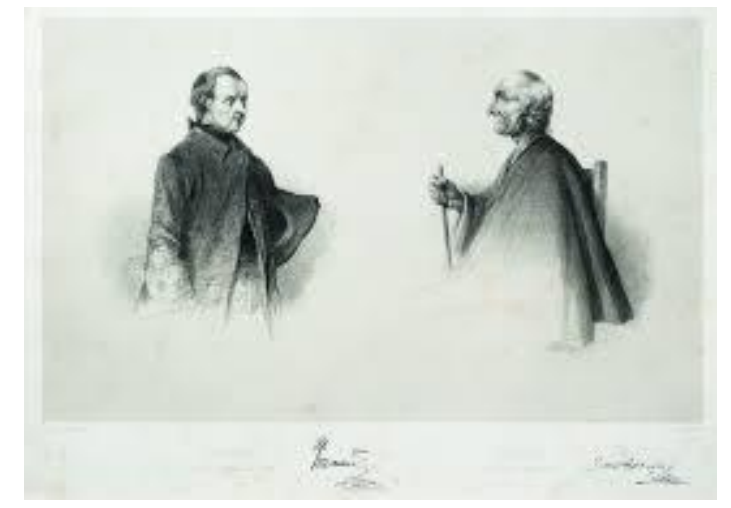

Fuente: Alfred Demersay. Histoire physique, économique et politique du Paraguay et des établissements jésuites. Paris: 1860 -1864.

Además de esta obra, el visitante francés produjo un grabado de la "maison habitée par Artigas a Ibiray" que publicaría en 1860 en una decena de litografías con motivos paraguayos. $^{33}$

¿Cómo transcurrió el encuentro entre el retratista y el modelo bajo las sombras del Yvyra-pytà, que rodeaban a la residencia de este último en el barrio de Trinidad? Demersay dejó anotado que "después de la muerte del dictador, se le permitió (a Artigas) establecerse en las cercanías de la Asunción. Es allí donde lo encontramos viviendo, como él mismo lo dijo, de la limosna del presidente López, alojado en Ybyray en una de sus casas; todavía recto y vigoroso a pesar de su gran edad". ${ }^{34}$ Está documentado que, en efecto, el Estado paraguayo le pagaba anualmente, con fondos del Tesorero de Guerra, una mesada para su manutención, aunque los montos variaron de manera sensible. Así, por ejemplo, en 1824 ascendía a 100 pesos, en 1828 figura que se le abonaban 90 pesos y en 1829 , tan solo 58 pesos. $^{35}$

Por lo demás, es probable que durante la confección del dibujo estuvieran presentes los dos ayudantes que Demersay menciona en la documentación dirigida al presidente López, como acompañantes imprescindibles en sus tareas en el Paraguay.

\footnotetext{
${ }^{32}$ Massimo Leone, "Rostros populares, rostros populistas: para una semiótica de la efigie heroica el caso de José Gervasio Artigas", en Edgardo Manero y Lucrecia Escudero Chauvel, con la colaboración de Juan Corvalán Espina (Coordinadores), Populismo(s). Intersecciones en las Ciencias Sociales (París, 2019), pp. 171-181.

${ }^{33}$ Las litografías forman parte, en la actualidad, del acervo de la Biblioteca Nacional del Paraguay. Véase Catálogo Colección Enrique Solano López. Biblioteca Nacional del Paraguay, Asunción, 2014.

34 Alfred Demersay, Histoire Physique, Économique, et Politique du Paraguay et des Établissements des Jesuites. Paris: Hachette, 1860 (1er. Tomo de textos), 1864 ( $2^{\circ}$ tomo) y un Atlas. La cita en página 366.

${ }^{35}$ Archivo Nacional de Asunción, Sección Civil y Judicial, Volumen 1329, Número 14, año 1836. Inventario de los útiles de la comandancia de San Isidro Labrador de Curuguaty al hacerse cargo Juan Manuel Gauto.
} 
Otros testimonios tangenciales nos aportan datos sobre el estado espiritual de los protagonistas del encuentro. Por ejemplo, se sabe que para la fecha de la cita con Demersay, Artigas ya había determinado que no regresaría a Uruguay y que todo lo que tenía que decir sobre su situación política ya se lo había confiado a su hijo José María, quien lo había visitado en 1846. A propósito, el escritor uruguayo Isidoro de María, casado con Sinforosa Navarrete Artigas (hija de Francisca, prima hermana de Artigas) registra que, en efecto, el hijo viajó a Asunción para ver y persuadir a su padre de que regresara a Uruguay, y que en conversaciones rodeadas de "confianza íntima", aquel habría manifestado que no se sentía con fuerzas para emprender el retorno. Agrega de María que aquella fue la primera vez que Artigas se desahogaba "expansivamente, después de 26 años de oscuridad y de silencio". ${ }^{36}$

Demersay bien podría haber ido al encuentro con una idea crítica sobre Artigas y sobre Francia, luego de recibir el testimonio del médico y botánico francés Aimé Bonpland en Santa Ana, Corrientes, de quien obtuvo, además, los apuntes personales que redactara durante sus años de confinamiento. Como se conoce, Bonpland fue apresado por orden de Francia en la localidad correntina en la que se hallaba bajo la protección de Francisco Ramírez, y donde se dedicaba a la formación de una colonia agrícola. El dictador paraguayo que había ordenado se respetara la vida del sabio, veía en esa fundación una amenaza a lo que consideraba la integridad patrimonial paraguaya. Conducido a Itapúa primero, y luego a San Borja, fue liberado en 1829. En 1837 se estableció en Santa Ana, en las inmediaciones de lo que es hoy Paso de los Libres; y allí lo encontró Demersay y recibió de su mano los apuntes que había redactado durante su cautiverio. ${ }^{37}$ También, se podría argumentar que la conversación entre Demersay y Artigas no modificó los conceptos que ya se habría formado el francés, influido por las lecturas de obras, muy críticas por cierto, sobre la situación paraguaya de la primera mitad del siglo diecinueve que se divulgaron en Europa antes de su viaje al Paraguay con fines científicos. Nos referimos, más precisamente, a los relatos de J. R. Rengger y M. Longchamp, Essai historique sur la révolution du Paraguay, et le gouvernement dictatorial $d u$ Docteur Francia, publicado en francés y en alemán en el año 1827, los textos de John Parish y de William Parish Robertson, Letters on Paraguay publicado en 1838, y Francia's Reign of Terror, en 1839.

Quince años después de su encuentro con Artigas, Demersay publicó en Paris la Histoire physique, économique et politique du Paraguay, en la que presentaba un panorama

\footnotetext{
${ }^{36}$ Vida del Brigadier General José Gervasio Artigas fundador de la Nacionalidad Oriental (Gualeguaychú: 1860), página 37.

${ }^{37}$ Guillermo Furlong, "Nuevos datos sobre Bonpland en Buenos Aires (1818)", en Anales de la Universidad del Salvador, $\mathrm{N}^{\mathrm{o}} 5$ (1969).
} 
negativo no sólo del gobierno de Francia, sino también de Carlos Antonio López y del propio caudillo oriental. En un plano especulativo se podría sostener que, no obstante haber mantenido alguna conversación sobre hechos o personajes concretos, no hubo nada en el encuentro con Artigas que creyó Demersay que merecía ser recogido en su obra, además del apunte sobre la limosna que recibía del Estado paraguayo. Por cierto, Laura Malosetti nos hace notar que el pensamiento crítico de Demersay sobre la condición y la actuación política de Artigas podría haber atravesado la confección del retrato. Sostiene que, desde la perspectiva del naturalista, el caudillo oriental era un anciano prisionero cuya fama de hombre carismático y peligroso perduraba. Prisionero, en primer lugar, del tiempo: la edad lo había vuelto un ser aparentemente inofensivo, sin dientes; pero también, de las condiciones miserables en que había vivido largos años. De modo que su retrato podría pensarse tanto como una prueba de su condición en aquel presente, como una indagación acerca de su pasado: el "único ojo visible, fijo en un punto, parece preservar algo del antiguo poder. En el resto de la figura el artista parece haber acentuado los signos de la decrepitud del viejo caudillo". 38

Todo indica que la propia experiencia en el Paraguay y la lectura de los apuntes de Bonpland incidieron directamente en las representaciones que sobre Artigas y Francia ofreció Demersay en su obra, así como sobre el sistema político de Carlos Antonio López, al que define de autocrático y sobre el que proyecta apenas una tenue imagen de su empuje progresista.

En el plano de los aportes documentados, hay que añadir que durante el tiempo que vivió en el Paraguay, Demersay confeccionó también un retrato de Francia. Al respecto, dejó explicado que lo había realizado tomando como modelo a la hermana del Supremo, doña Petrona, que lo había sobrevivido. De este modo circuló la primera imagen que se conserva de Francia, pero con los rasgos de su hermana. Se trataba, claro está, de una invención, puesto que no existe ningún dibujo al natural del Supremo, ni siquiera de memoria. Demersay decidió publicarlo junto al de Artigas en el Atlas que formó parte de la Histoire du Paraguay. Parecen mirarse uno a otro, como poniendo en escena el recelo de Francia hacia el antiguo enemigo. Debajo de cada uno, sus nombres, sus fechas de nacimiento y muerte, y sus firmas. La de Artigas, muy temblorosa, la de un anciano que durante muchos años probablemente apenas hubiera empuñado una pluma. ${ }^{39}$ De modo que, el escaso testimonio que legó acerca

\footnotetext{
${ }^{38}$ Laura Malosetti Costa, "El primer retrato de Artigas: un modelo para deconstruir" ..., p. 6.

${ }^{39}$ Casi una década antes de la publicación del Atlas, Alfred Demersay publicó en Paris un pequeño folleto de 16 páginas titulado Le docteur Francia; dictateur du Paraguay (Paris: Henry Plon, 1856).
} 
del encuentro con Artigas, el retrato que fue el fruto de ese breve intercambio, parecen unificarse en el propósito intelectual de Demersay de dar a conocer sus propias ideas en Europa sobre el Paraguay, sobre su historia en general, y sobre el gobierno de Carlos Antonio López en particular.

\section{Referencias bibliográficas}

\section{Fuentes de Archivo}

Archivo Nacional de Asunción, Sección Civil y Judicial, Volumen 1329, Número 14, año 1836. Inventario de los útiles de la comandancia de San Isidro Labrador de Curuguaty al hacerse cargo Juan Manuel Gauto.

Archivo Nacional de Asunción, Colección Historia de la República del Paraguay (ex Colección Río Branco): Py 443- 1-12.

\section{Bibliografía}

Baratta, María Victoria. "Paraguay en las crónicas de los viajeros durante el gobierno de José Gaspar Rodríguez de Francia” en Revista Paraguay desde las Ciencias Sociales, revista del Grupo de Estudios Sociales sobre Paraguay, nº 9 (2018): pp. 23-43.

Biblioteca Nacional del Paraguay. Catálogo Colección Enrique Solano López. Asunción: 2014.

Demersay, Alfred. Histoire Physique, Économique, et Politique du Paraguay et des Établissements des Jesuites. Paris: Hachette, 1860 (1er. tomo), 1864 (2ºmo).

Furlong, Guillermo. "Nuevos datos sobre Bonpland en Buenos Aires (1818)", en Anales de la Universidad del Salvador, № 5 (1969).

Leone, Massimo. "Rostros populares, rostros populistas: para una semiótica de la efigie heroica el caso de José Gervasio Artigas”. En Edgardo Manero y Lucrecia Escudero Chauvel, con la colaboración de Juan Corvalán Espina (Coordinadores), Populismo(s). Intersecciones en las Ciencias Sociales. París: 2019.

Malosetti Costa, Laura. "El primer retrato de Artigas: un modelo para deconstruir", En Caiana. Revista de Historia del Arte y Cultura Visual del Centro Argentino de Investigadores de Arte (CAIA), No 3 (2013): pp. 1-14. 